\title{
Multiaxial High Cycle Fretting Fatigue
}

\author{
José Alexander Araújo ${ }^{1}$, Gabriel Magalhães Juvenal Almeida ${ }^{1}$, Fábio Comes Castro ${ }^{1}$ and \\ Raphael Araújo Cardoso ${ }^{1}$ \\ ${ }^{1}$ Department of Mechanical Engineering, University of Brasilia, Brasilia, DF 70910-900, Brazil
}

\begin{abstract}
The aim of this work is to show that multiaxial fatigue can be successfully adpted to model fretting problems. For instance, the paper presents (i) the critical direction method, as an alternative to the critical plane concept, to model the crack initiation path under fretting conditions and (ii) studies on size effects considering the influence of incorporating fretting wear on the life estimation. A wide range of new data generated by a two actuators fretting fatigue rig considering $\mathrm{Al} 7050-\mathrm{T} 7451$ and of Ti-6Al-4V aeronautical alloys is produced to validate these analyses. It is shown that, the development of appropriate tools and techniques to incorporate the particularities of the fretting phenomenon into the multiaxial fatigue problem allow an accurate estimate of the fretting fatigue resistance/life in the medium high cycle regime. Such tools and techniques can be extended to the design of other mechanical components under similar stress enviroments.
\end{abstract}

\section{Introduction}

Fretting fatigue is a type of failure that may occur in components of a mechanical assembly, which are not only subjected to some sort of vibration, but also to a cyclic varying remote stress [1]. In these cases, a superficial damage arises due to a minute relative displacement between the contacting surfaces. Further, the normal and shear contact loads generate high superficial stresses that nevertheless fall rapidly with distance from the contact interface. Crack initiation in this type of problem is thus mainly governed by the stress field underneath the contact, however the danger of these cracks to progress to a catastrophic failure is associated with the severity of the bulk fatigue stress. The role of the mild superficial damage generated by the micro slip of the contacting surfaces on crack initiation is still a matter of intense debate in the academic scenario [1-13] and will certainly be addressed here as well. Clearly, fretting fatigue is a quite complex phenomenon as it involves tribological issues, multiaxial non-proportional stresses, high stress gradients, etc. The aim of this work is to show that multiaxial fatigue approaches can be successfully used to model fretting problems. In this setting, a review of recent developments carried out at the University of Brasília on the use of multiaxial non-local approaches to estimate high cycle fretting fatigue damage will be presented. More specifically, the work will address the techniques we have considered to incorporate the effect of non-proportional loading and stress gradient effects in the modelling. A number of these techniques represent innovative contributions in the field of plain multiaxial fatigue [14-18], hence, they can easily be extended to estimate fatigue strength or life not only under fretting conditions, but also to problems with similar mechanical features, 
such as in notched components under complex loading histories. For instance, the paper presents the critical direction method [17-19], as an alternative to the critical plane concept, to model the crack initiation path under fretting conditions in an Al 7050-T7451 aeronautical alloy [18-20]. A large battery of fretting data containing new and recently published data is made available to validate this proposal. Further, new studies and data on size effects considering the influence of incorporating fretting wear effects on the life estimation of Ti$6 \mathrm{Al}-4 \mathrm{~V}$ are presented [13]. Last but not least, the complex experimental facilities and techniques required to produce the fretting fatigue data used to validate the analyses are presented and discussed [12,13]. It is shown that, the development of appropriate tools and techniques to incorporate the particularities of the fretting phenomenon into the multiaxial fatigue problem allow an accurate estimate of the fretting fatigue resistance/life in the medium high cycle regime.

\section{Recent developments}

\subsection{Theory of Critical Distances (TCD)}

It is well known that hot spot approaches overestimate fatigue damage assessment when geometrical discontinuities generating high stress gradients are present (e.g. sharp notches and cracks) [21]. Moreover, hot spot approaches are not well suited for fretting problems either [22]. In this case, contact loads are generally responsible for high levels of stress concentration near the contact zones where it is highly influenced by the contacting geometries. Even though the Theory of Critical Distances (TCD) [21, 23] has been first proposed to deal with stress raisers such as notches and cracks, over the last years, it has also been used to evaluate fretting fatigue problems $[5,12,15]$. In its most fundamental definition, the idea consists in evaluating a fatigue criterion $F(\boldsymbol{\sigma})$ inside a process volume $V$ surrounding a stress raiser and compare it with $F_{c r i t}$, a material property depending on the multiaxial fatigue criterion used and obtained from a smooth specimen under fatigue limit conditions. The volume $V$ is generally associated with a material parameter given by $[21,23]$ :

$$
L=\frac{1}{\pi}\left(\frac{\Delta K_{t h}}{\Delta \sigma_{-1}}\right)^{2}
$$

where $\Delta K_{t h}$ is the material threshold stress intensity factor range for long cracks and $\Delta \sigma_{-1}$ is the uniaxial fatigue limit range for fully reversed axial loading conditions. For 2D analysis, the TCD can be expressed in its simplified versions considering an area, a line or even a point [21]. For example, in the Point Method [23], fatigue endurance occurs for:

$$
F(\sigma(L / 2)) \leq F_{\text {crit }}
$$

Castro et al. [15] considering the Point Method have shown that, the material characteristic length given by Eq. (2) depends on the multiaxial fatigue considered. In this setting, it can be obtained through the results of two different fatigue tests, one of them considering a smooth specimen and the other a cracked specimen under threshold conditions (i.e. non-propagating crack, $\Delta K_{I}=\Delta_{t h}$ ), Fig. 1 . 


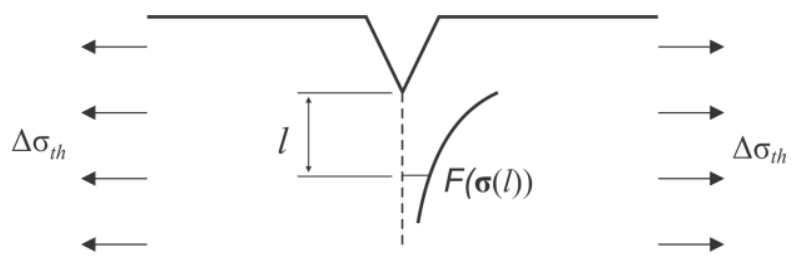

Fig. 1. Cracked body subjected to fully reversed uniaxial loading with $\Delta \sigma_{t h}$ being the stress range at threshold conditions.

In this case, for the cracked body, stress solutions can be recovered through LEFM for all time instants $t$ :

$$
\boldsymbol{\sigma}(t)=\frac{1}{2} \frac{\Delta K_{I}}{\sqrt{2 \pi l}} \sin (\omega t)\left(\boldsymbol{e}_{x} \otimes \boldsymbol{e}_{x}+\boldsymbol{e}_{y} \otimes \boldsymbol{e}_{y}\right)
$$

where $\omega$ is the angular frequency of the nominal fatigue stress and $l$ is the vertical distance from the crack tip, Fig. 1 . In order to determine $l$ for a given multiaxial fatigue criterion, $F$, one has first to evaluate it considering the smooth specimen under fatigue limit conditions. Then one has to compare it with the one obtained considering the stress state provided by the cracked specimen under threshold considitons (Eq. (4) with $\Delta K_{I}=\Delta_{t h}$ ). In this case, when considering the MWCM for instance, it can be shown that $l$ is half of $L$ [15], which coincides with Taylor's previous definition [21], where the author assumes that $l$ is the same no matter the fatigue criterion assessed. On the other hand, when regarding the stress invariant-based criterion proposed by Crossland, this distance becomes dependent on the material fatigue limits considering two different stress states [15].

\subsection{Multiaxial Fatigue}

A brief descripion of the multiaxial fatigue criteria which will be considered in this work for the proposed assessment procedures is now provided.

\subsubsection{Fatemi-Socie}

The celebrated Fatemi and Socie [24] multiaxial fatigue parameter (FS) is usually considered to evaluate the crack initiation risk in material and loading conditions where the fatigue damage is mainly driven by shear stresses. It can be expressed in (elastic) stress terms as:

$$
\mathrm{FS}=\tau_{a}\left(1-K \frac{\sigma_{n, \max }}{\sigma_{\mathrm{y}}}\right)
$$

where $\tau_{a}$ is the shear stress amplitude, $K$ is a material constant, $\sigma_{n, \max }$ is the maximum normal stress over a loading cycle, and $\sigma_{\mathrm{y}}$ is the material yield stress. In the formulation of this parameter, the response of the material to normal stresses is adjusted by the $K / \sigma_{\mathrm{y}}$ ratio. Altough in its original formulation [24] the critical plane was defined as the one where $\tau_{a}$ reached its maximum value, usually it has been defined as the plane over which the scalar FS is maximum, i.e., the plane where Eq. (4) is maximized.

\subsubsection{Smith-Watson-Topper}


The Smith, Watson and Topper criterion (SWT) [25] is a critical plane model conceived for materials and configurations where the crack initiation mechanism is predominantly governed by normal stresses (or normal strains).

$$
\mathrm{SWT}=\sigma_{n, \max } \frac{\Delta \varepsilon_{n}}{2}
$$

where $\sigma_{n, \max }$ and $\Delta \varepsilon_{n}$ are the maximum normal stress and normal strain range on the material plane that maximizes SWT .

\subsubsection{Modified Whöler Curve Method}

The Modified Whöler Curve Method (MWCM) [26] is also used to estimate fatigue resistance or life under complex loadings. It can be writen as:

$$
\operatorname{MWCM}=\tau_{\mathrm{a}}+\kappa \frac{\sigma_{n, \max }}{\tau_{a}}
$$

where $\kappa$ is a fatigue material property. Here the critical plane is the one where $\tau_{\mathrm{a}}$ is maximum. If more than one plane experiences the same value of maximum $\tau_{a}$, the critical plane will be the one where $\sigma_{n, \max }$ is the highest.

\subsubsection{Findley}

Findley's [27] multiaxial criterion is among the first to use the concept of a preferential (critical) material plane for crack initiation:

$$
\mathrm{FP}=\left(\tau_{a}+\gamma \sigma_{n, \max }\right)_{\max }
$$

where $\gamma$ is a material parameter. This model assumes that shear stresses are responsible for crack initiation but also realizes that normal stresses to the plane increase crack opening and thus the rate of initial growth. The critical plane is defined as the one that maximizes Findley's parameterFP.

\subsubsection{Maximum Rectangular Hull}

Note that, all the other multiaxial criteria but the SWT demand the computation of an equivalent shear stress amplitude, $\tau_{a}$. In addition, all the models require the use of the maximum normal stress $\sigma_{n, \max }$. The computation of $\sigma_{n \text {,max }}$ is straightforward. On the other hand, the definition of the shear stress amplitude, $\tau_{a}$, is more challenging once the shear stress vector on a material plane, $\boldsymbol{\tau}$, may describe a generic path $\psi$ over time, Fig. 2. Although there are many methods for the computation of $\tau_{a}$ [14-16], one of the most popular is the Minimum Circumscribed Circle (MCC) [28]. However, the MCC possesses two main drawbacks. One of them is that its solution requires elaborate algorithms (min-max optimization problem). The other comes from the fact that this method is not sensitive to the non-proportionality of the shear stress path, Fig. 2(a), which may lead to inaccurate measurements of the fatigue damage [16].

An alternative method to compute $\tau_{a}$ was proposed by Mamyia and Araújo and coworkers $[14,16]$. These authors claimed that the equivalent shear stress amplitude, $\tau_{a}$, can be obtained by means of a Maximum Rectangular Hull (MRH) enclosing the stress path $\psi$ on the material plane $\Delta$, Fig. 2(b). In this case, for each $\beta$-oriented rectangular hull, a certain 
shear stress amplitude, $\tau_{a}(\beta)$, is found. At the end, the equivalent shear stress amplitude is defined by maximizing $\tau(\beta)$.

a)

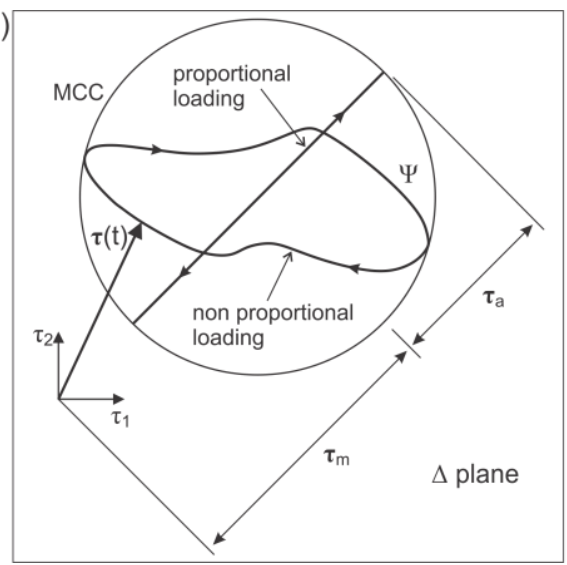

b)

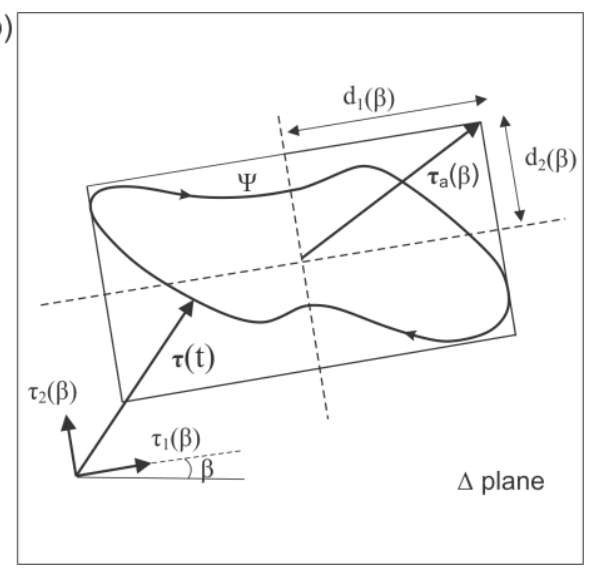

Fig. 2. Schematic representations of (a) the MCC and (b) the MRH for a given shear stress path $\psi$.

Beside its simplicity by means of implementation, the MRH is sensitive to proportional and non-proportional shear stress paths. In [16] it is also verified that fatigue estimates provided by the MWCM are more accurate when $\tau_{a}$ is computed via the MRH rather than the MCC.

\subsection{Critical Direction Method (CDM)}

The concept of a preferential critical plane for crack initiation based on the evaluation of normal and shear stress components in a single material point holds, from a mechanical point of view, when the component is under a uniform stress field. For rapidly varying stress fields, as it is the case in fretting fatigue, it is unlikely that a unique material plane orientation obtained from the most serverely stressed material point will define the crack orientation along some process zone. In this setting, Araújo and co-workers [17-19] proposed the so called Critical Direction Method (CDM) to estimate fretting crack initiation direction. The underlying concept of the CDM is that crack direction in its early stages will also be defined by an average state of stress within a certain zone (or length). Thus it requires the search for a $\theta$ oriented line/plane emanating from the crack initiation point (hot spot) whose length is $2 L$. After discretization of such line in many material points along $r$, the time varying average normal and shear stresses, $\bar{\sigma}_{n}$ and $\bar{\tau}$, are calculated as:

$$
\bar{\sigma}_{n}(\theta, t)=\frac{1}{2 L} \int_{0}^{2 L} \sigma_{n}(r, \theta, t) d r
$$

and

$$
\bar{\tau}(\theta, t)=\frac{1}{2 L} \int_{0}^{2 L} \tau_{n}(r, \theta, t) d r
$$

For a complete fatigue cycle, the average values of the maximum normal stress, $\bar{\sigma}_{n, \max }(\theta)$, the normal stress amplitude, $\bar{\sigma}_{n, a}(\theta)$ and the shear stress amplitude, $\bar{\tau}_{a}(\theta)$, associated with 
each $\theta$ direction can be found and used in a multiaxial fatigue criteria. For more details about the CDM, the reader is referred to the Ref. $[17,18]$.

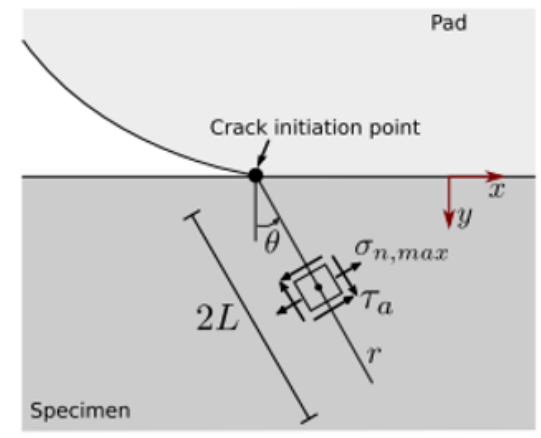

Fig. 3. Illustration of the Critical Direction Method. [18].

\subsection{Effect of modelling wear}

Most of the work done so far concerning fretting fatigue life estimates neglect wear when partial slip conditions are observed [1-5]. According to classical results as the fretting map presented by Vingsbo and Söderberg [2], wear seems to have a detrimental effect in terms of fatigue life for partial slip conditions and a beneficial one when gross slip takes place. In the latter, material loss is high and cracks are worn out before they even start propagating. Recently, some numerical investigations have somehow confirmed these aspects [6-10].

To model wear, either a local version of Archard's law [6,7] or the dissipated friction energy relation are used $[10,11]$. The main advantage of the latter is that it accounts easily for changes in the friction coefficient along the contact surface. In addition, both aforementioned approaches can be readily implemented on a nodal basis in FEA. In this case, for example, when considering the dissipated energy relation, vertical displacement of the contact nodes representing the material loss can be obtained by:

$$
\Delta h_{i, j}=\sum_{k=1}^{n_{i n c}} \alpha q\left(x_{j}, t_{k}\right) \Delta s\left(x_{j}, t_{k}\right) \Delta N
$$

where $\Delta h_{i, j}$ is the increment of wear for given node $j$ belonging to the contacting surface at the simulation of the $i^{t h}$ fretting cycle, $\alpha$ is the dissipated energy wear coefficient while $q\left(x_{j}, t_{k}\right)$ and $\Delta s\left(x_{j}, t_{k}\right)$ are, respectively, the contact shear traction and the relative slip increment of the contact node located at the position $x_{j}$ for the time instant $t_{k}$. Note as well that, in Eq. (10), there is a jumping factor $\Delta N$ amplifying wear computations. This is a numerical strategy used to speed up simulations [6-10], where in this case, wear is assumed to be constant for a given number of cycles $\Delta N$. In order to update contacting surfaces over the simulation, either remeshing [6,7,10] or adaptive meshing techniques [8,9] may be considered.

It worth noting that, when wear effects are considered in the analysis, contact subsurface stresses are constantly changing. In this case, multiaxial fatigue parameters cannot be readily applied to the problem. One of the most common ways of overcoming this issue is by using an incremental damage formulation $[8,10]$. In this setting, the simplest one is the Miner's linear damage rule. 


$$
D_{n}=\sum_{i=1}^{n} \frac{\Delta N}{N_{f, i}}
$$

where $D_{n}$ is the cumulated damage for the $n^{\text {th }}$ fretting cycle simulated (i.e. $n \times \Delta N$ wear cycles considered) and $N_{f, i}$ is the fatigue life estimated for a given stress state at the $i^{t h}$ simulated fretting cycle.

\section{Experimental data}

\subsection{Two actuators fretting fatigue rig at the University of Brasilia (UnB)}

The fatigue team at the University of Brasília $(\mathrm{UnB})$ has recently designed and mounted a quite interesting two vertical actuators fretting rig [12,18-20]. This machine is capable to apply and independently control time varying bulk and fretting loadings. The normal contact load is static and applied by a simpler hydraulic system connected to an accumulator and a manual pump. A schematic view of the test rig is shown in Fig. 4.

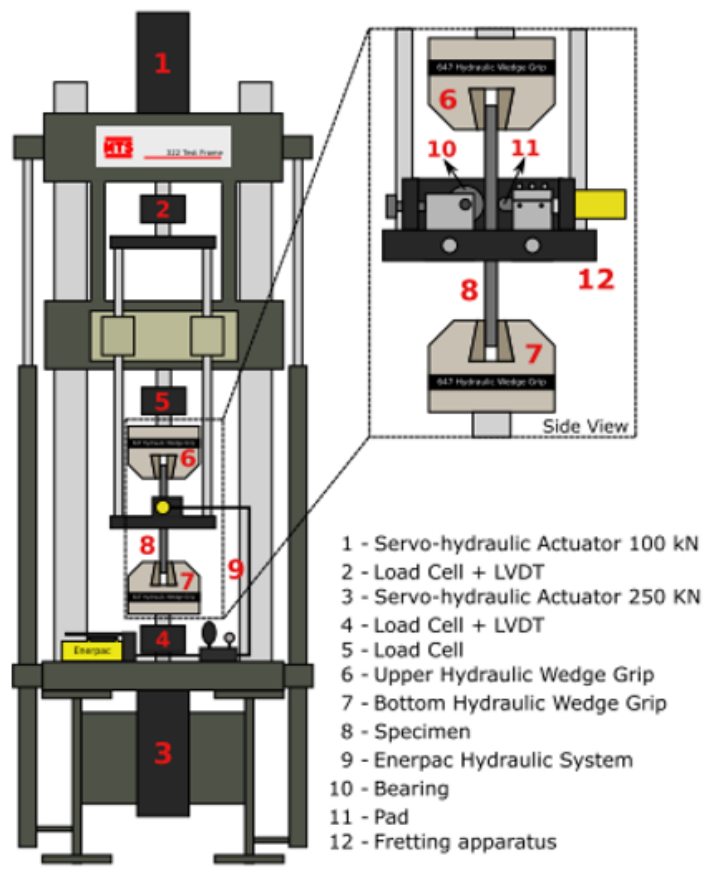

Fig. 4. Layout of UnB's Two Actuators Fretting Fatigue Rig.

The fretting fatigue apparatus, whose details are shown in the amplified scheme of the side view within Fig. 4, is mounted on the MTS frame. Such apparatus holds the pads carriage and the Enerpac static actuators $(50 \mathrm{kN})$, which keep the pressure on the pads. It can also operate using one pad and a bearing holder, as depicted. Four mettalic rods connect the pad carriage to the upper $100 \mathrm{kN}$ hydraulic actuator (numbered as 1) and its load cell (numbered as 2). This actuator provides the tangential forces at the fretting contact. The $250 \mathrm{kN}$ lower actuator (3) applies the bulk fatigue force to the specimen. 
Several fretting tests under partial slip conditions were carried out using the aforementioned fretting fatigue rig [18]. These tests aimed to evaluate the influence of the mean bulk stress $\left(\sigma_{b, m}\right)$, the tangential load amplitude $\left(Q_{a}\right)$, and the pad radius $(R)$ on the crack initiation path. The experimental configuration involved cylindrical pads pressed against flat dog-bone specimens, both made of Al7050-T7451 alloy. Eight set of tests were carried out. For the load configurations 1 to 3, tangential load amplitude was varied, while all other loading conditions were kept constant (including pad's radii). To assess the influence of the pad radius (load configuration 4 and 5), two different size of cylindrical pads were chosen, while all other parameters were held the same. In these specific tests, the normal and tangential loads were specially designed to keep the ratio $Q / P$ and the peak pressure $\left(p_{0}\right)$ equal. Finally, in the load configurations 6 to 8 , only the mean bulk stress was varied. Measurements of crack initiation path were taken and their average values are depicted in Fig. 7, together with the estimates provided by the CDM. The measurement procedure to obtain an average orientation consisted in locating the initiation site of the dominant crack and then drawing the best straight line of length $2 L$ that could describe it, as shown in Fig. 5. This size was chosen as different works [21,23] have proved that, when averaging procedures over unidimensional values are required, the best estimates of fatigue strenght/life are obtained considering a line of length $2 L$.

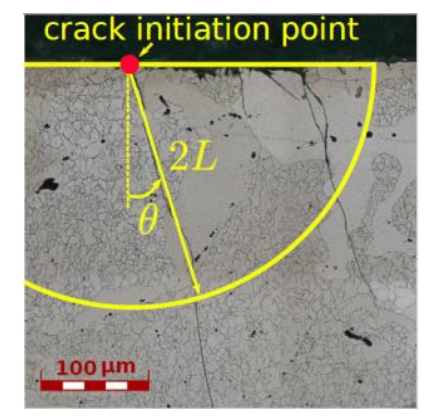

Fig. 5. Measurement of fretting crack orientation considering the CDM.

\subsection{Group-2 tests (size effect on fretting fatigue)}

This group of tests aimed to investigate size effects on fretting fatigue [12]. In order to do so, two sets of tests were designed. In one of them, Group-2a tests, the aim was to investigate the influence, on fretting fatigue life, of the volume of material being stressed beneath the contact surface. In this case, pairs of tests were designed under partial slip conditions so that they had the same stress gradient close to the contact surface, the same slip (damaged) area and different volumes of material being stressed under the contact region, Fig. 6(a). In order to ensure the same slip area for contacts with different widths ( 8 and $13 \mathrm{~mm})$ that generated different volumes under the contact, the amplitude of the tangential load applied to the cylindrical pads were different. The same stress gradient for a pair of tests was secured by carefully choice of the bulk fatigue loads applied to the specimens. On the other hand, in an attempt to verify the influence of the slip areas on fretting fatigue life, another set of tests, named Group-2b tests, was designed. In this case, pairs of testes were proposed so that the stress gradient close to the contact surfaces were the same but now the sizes of the slip areas were different, Fig. 6(b). Such condition is easily obtained by only changing specimens' thickness while keeping the same bulk load and contact loads per unity of length. In order to enforce the same stress gradient nearby the contact edges for given a pair of tests, Araújo et al. [12] have used the TCD in terms of the Point Method in association with the MWCM. For 
more details about the loadings determination, pads and specimens specifications, the reader is referred to [12]. The material considered in their analyses was the TI-6Al-4V.

In all Group 2 tests, the normal load, $P$, per unity of length applied to cylindrical pads was the same, which implied in contact zones with the same size for all the tests. All testes were carried out under partial slip conditions. The contact configuration considered in the analyses was the cylinder-on-plane one. A pad radius of $70 \mathrm{~mm}$ was the same for all the tests. A schematic view of geometry and loading conditions assessed in the experiments are depicted in Fig. 8.

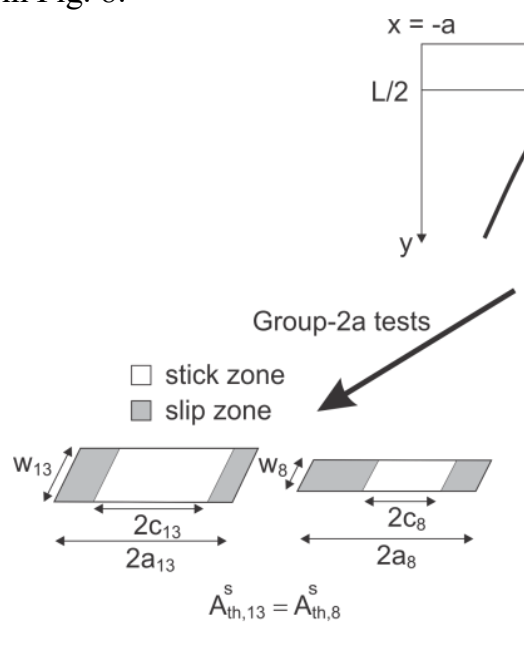

(a)
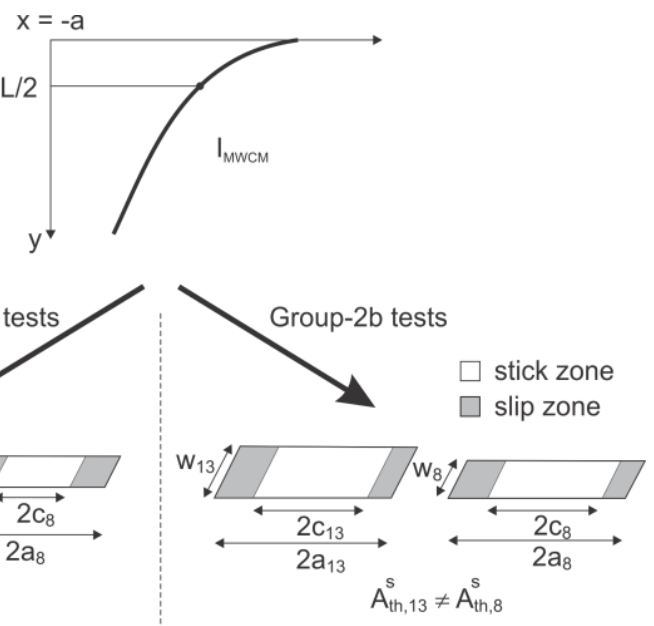

(b)

Fig. 6. Schematic representation of the Group-2 tests: (a) Group-2a tests with the same slip areas for different widths, (b) Group-2b tests with different slip zone zones.

\section{Results and Discussions}

\subsection{Loading effects on fretting crack initiation path (Group 1 tests)}

Almeida et al. [18] and Vantadori et al. [19] combined the Critical Direction Method with multiaxial criteria to estimate early crack orientation in fretting fatigue. While [18] considered the FS, SWT and the MCWM criteria in their analysis, [19] used the Carpinteri et al. criterion [30]. Group 1 tests were used to validate the analysis. Results of these works are summarized in Fig. 7. From the experimental point of view, it is possible to obverse an increase of the average values of crack initiation direction with the raise of the tangential or the bulk load. The effect of increasing the pad radius on the early fretting crack direction was not significant. In addition, Fig. 7 also depicts the numerical estimates when the CDM is associeted with these four different multiaxial fatigue criteria. It can be clearly seen that the best results for crack initiation path were obtained by the combination of the CDM with the SWT and Carpinteri et al. multiaxial fatigue criteria, while the FS criterion acossiated with the CDM provided the least acurate etimates. According to this model, fretting cracks would initiate outward the contact zone moving away from the contact region which completely differs from experimental data. 


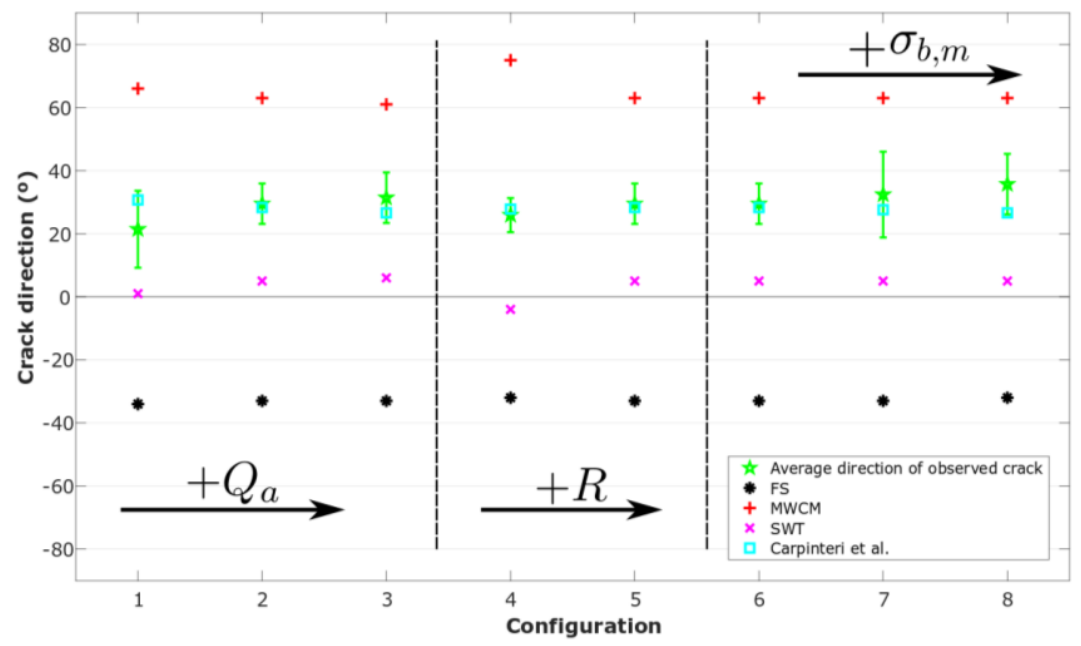

Fig. 7. Observed versus predicted crack orientation based on the Critical Direction Method. Results for FS, MWCM, SWT and Carpinteri et al. [18,19].

\subsection{Evaluation of wear effects on fretting fatigue life estimates (Group 2 tests)}

Recently, Cardoso et al. [13] have considered Group-2 tests in order to investigate the influence of wear on fretting fatigue life estimates under partial slip conditions. These works intended to verify if the simplifying approach of neglecting wear commonly adopted in the fretting field $[3-5,22]$ is well suited when partial slip conditions are predominant. In this case, life estimates considering wear were compared to both, life estimates neglecting wear and experimental observations.

The wear modelling process was carried out by means of an incremental FE framework. The vertical displacement of the contact nodes representing the material loss due to wear was obtained via Eq. (10) and contacting surfaces were updated by using a remeshing technique. As contact subsurface stresses constantly change due to wear, Miner's rule was applied to predict failure, Eq. (11) $(D=1)$. During the analysis, damage computations were performed on the centroid of the elements nearby the contact surface. The FE model (ABAQUS) is depicted in Fig. 8 as well as the loading conditions considered in their analyses.

To estimate life we considered the SWT, Findley's and MWCM criteria. These models were calibrated with uniaxial data for the Ti-6Al-4V provided by [29]. Neglecting wear, life could be directly estimated by evaluating the multiaxial criteria at the center of the process zone (point method), which is $L / 2$ vertically distant from the contact trailing edge (hot spot). On the other hand, when wear takes place, Miner's rule is also required. Besides, the hot spot point at the contact surface was not fixed over the fretting cycles. In this case, the strategy adopted in [13] was to cumulate increments of Miner's damage parameter but only considering points at least $L / 2$ vertically distant from the contact surface. Note that in both approaches, neglecting or considering wear, the TCD was used to account for stress gradient effects inherent of contact problems. In Figure 9, life estimates [13] considering and disregarding wear effects are compared to the actual ones (Group-2 tests Subsection 3.3). Circle markers represent results obtained when the SWT fatigue parameter is used. As can be seen, both approaches lead to estimates within a factor of two. It can also be observed that results accounting wear are slightly less disperse. In addition, when considering wear, the SWT parameter was less conservative compared to the other two shear stress based models. When neglecting wear effects, such patterns were hard to be drawn. 

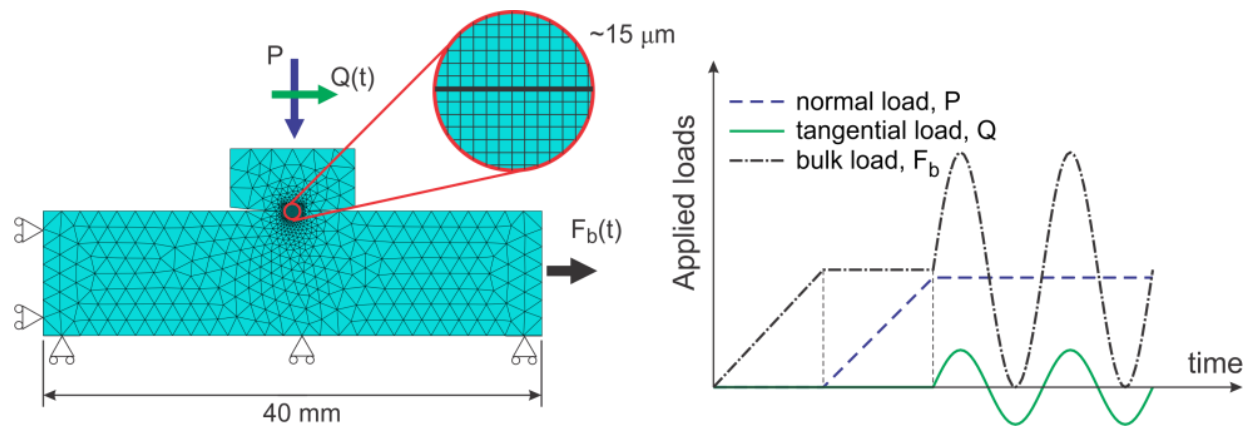

Fig. 8. FE model considered in the life estimate analysis by [13].

At the end, for the data assessed in [12] and in [13], i.e. fretting fatigue tests performed on a Ti-6Al-4V under partial slip regime, it seems that the conventional approaches for evaluating fretting fatigue problems, where wear is neglected, already provide satisfactory results. Note as well that, the slight improvement obtained when simulating wear may not be justified by its high computational cost when compared to simulations where wear is disregarded. However, it is worth remarking that, when neglecting wear, failure is systematically predicted at the contact trailing edge, while including it in the analysis, failure is observed towards the stick/slip zone transition.

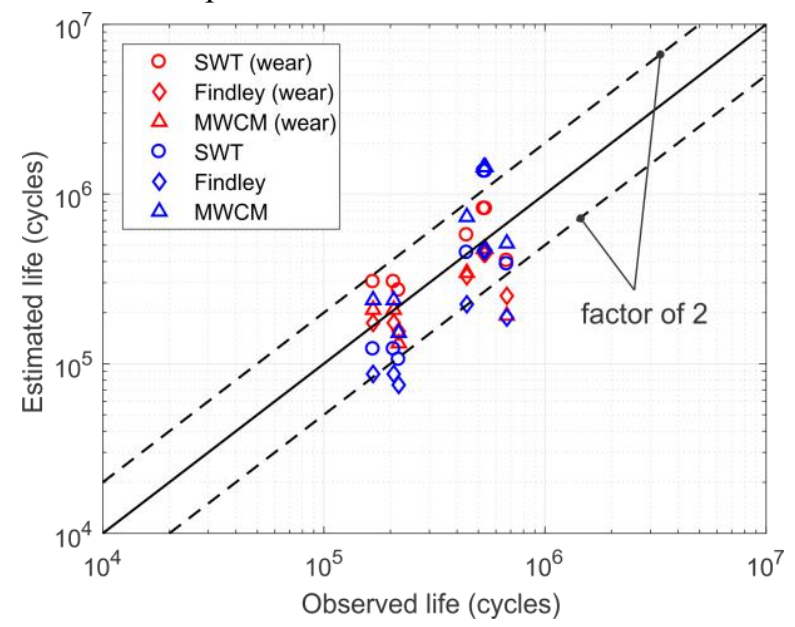

Fig. 9. Estimated vs observed fretting fatigue lives reported in $[12,13]$.

\section{Conclusion and perspectives}

This work has shown that the development of appropriate tools and techniques to incorporate the particularities of the fretting phenomenon into the multiaxial fatigue analyses allow accurate estimates of the fretting fatigue resistance/life and early crack orientation in the medium high cycle fatigue regime. Further, a wide range of new data considering tests on Al 7050-T7451 and of Ti-6Al-4V aeronautical alloys was generated to validate these analyses. Such data was produced by an innovative two independent vertical actuators fretting fatigue rig mounted at the University of Brasilia. More specifically the following conclusions can be drawn from the present work:

- For non-proportional stress histories the Maximum Rectangular Hull (MRH) should be considered to computer the amplitude of the shear stress vector projected in the critical plane; 
- for problems involving high stress gradients, such as in fretting fatigue, the critical plane concept, which is associated with the orientation of a plane passing through a single point in a material under uniform stress distribution, does not provide satisfactory results for crack initiation orientation. On the other hand, the CDM concept applied to the multiaxial models not only provides better estimates for the crack initiation path in fretting fatigue, but it is also mechanically more consistent;

- life estimates methodologies taking into account the contact/stress evolution due to material removal and life estimates where these effects are neglected (notch analogue approach) were proposed and assessed against data for a Ti6Al-4V alloy tested under partial slip conditions;

- For this Ti-6Al-4V alloy tested under partial slip conditions the accuracy of life predictions was not significanlty improved by including the wear effects. On the other hand, in these cases, computational cost can be substantially increased, mainly if we are dealing with complex real components.Note that many fretting cycles need to be evaluated when simulating wear, which is not the case when it is disregarded. In this case, only the information from one cycle is needed.

\section{References}

1. J. A. Araújo, D. Nowell, The effect of rapidly varying contact stress fields on fretting fatigue, Int. J. Fatigue, 24, 763-775, (2002)

2. O. Vingsbo, S. Söderberg, On fretting maps, Wear, 126, 131-147 (1988)

3. T. Hattori, M. Nakamura, T. Watanabe, Simulation of fretting-fatigue life by using stress-singularity parameters and fracture mechanics, Tribology International, 36: 8797 (2003)

4. C. Navarro, S. Muñoz, J. Dominguez, On the use of multiaxial fatigue criteria for fretting fatigue life assessment, Int. J. Fatigue, 30, 32-44 (2008)

5. J. A. Araújo, L. Susmel, M. S. T. Pires, F. C. Castro, A multiaxial stressbased critical distance methodology to estimate fretting fatigue life, 108, 2-7 (2017)

6. I. R. McColl, J. Ding, S. B. Leen, Finite element simulation and experimental validation of fretting wear, Wear, 256, 1114-1127 (2004)

7. J. Ding, S. B. Leen, I. R. McColl, The effect of slip regime on fretting wear-induced stress evolution Int. J. Fatigue, 26, 521-531 (2004)

8. J. J. Madge, S. B. Leen, P. H. Shipway, Contact-evolution based prediction of fretting fatigue life: effect of slip amplitude, Wear, 263, 542-551 (2007)

9. A. Cruzado, S. B. Leen, M. A. Urchegui, X. Gómez, Finite element simulation of fretting wear and fatigue in thin steel wires, Int. J. Fatigue, 55, 7-21 (2013)

10. S. Garcin, S. Fouvry, S. Heredia, A fem fretting map modeling: Effect of surface wear on crack nucleation, Wear, 330, 145-159 (2015)

11. S. Fouvry, T. Liskiewicz, T. Kapsa, S. Hannel, E. Sauger, An energy description of wear mechanisms and its applications to oscillating sliding contacts, Wear, 255, 287-298 (2003)

12. J. A. Araújo, B. Ferry, C. Montebello, J. Meriaux, S. Pommier, Studies of size effects in fretting fatigue, Tribol. Int, (submmitted for publication)

13. R. A. Cardoso, T. Doca, D, Néron, S. Pommier, J. A. Araújo, Wear numerical assessment for partial slip fretting fatigue conditions. Tribol. Int (submmitted for publication) 
14. E. N. Mamiya, J. A. Araújo, F. C. Castro, Prismatic hull: a new measure of shear stress amplitude in multiaxial high cycle fatigue, Int. J. Fatigue, 31, 1144-1153, (2009)

15. F. C. Castro, J. A. Araújo, N. Zouain, On the application of multiaxial high-cycle fatigue criteria using the theory of critical distances. Engng. Fract. Mech, 76, 512-524, (2009).

16. J. A. Araújo, A. P. Dantas, F. C. Castro, E. N. Mamiya, J. L. A. Ferreira, On the characterization of the critical plane with a simple and fast alternative measure of the shear stress amplitude in multiaxial fatigue,Int. J. Fatigue, 32, 1092-1100, (2011)

17. R. A. Cardoso, J. A. Araújo, J. L. A. Ferreira, F. C. Castro, Crack path simulation for cylindrical contact under fretting conditions, ,Frattura ed Integrità Structurale, 35, 405 413, (2016)

18. G. M. J. Almeida, F. C. Castro, Cardoso, J. A. Araújo, R. A., Investigation of early crack orientation in Al 7050-T7451 under fretting conditions. Tribol. Int (submmitted for publication), (2019)

19. S. Vantadori, G. M. J. Almeida, G. Fortese, G. C. V. Pessoa, J. A. Araújo, Early fretting crack orientation by using the critical plane approach, Int. J. Fatigue, 114 (2018)

20. J. A. Araújo, G. M. J. Almeida, J. L. A. Ferrreira, C. R. M. da Silva, F. C. Castro, Early cracking orientation under high stress gradients: The fretting case, Int. J. Fatigue, 100 (2017)

21. D. Taylor, , Geometrical effects in fatigue: a unifying theoretical model, Int. J. Fatigue, 21, 413-420, (1999)

22. J. A. Araújo, L. Susmel, D. Taylor, J. C. T. Ferro, J. L. A. Ferreira, On the prediction of high-cycle fretting fatigue strength: Theory of critical distances vs. hot-spot approach Engng. Fract. Mech, 75, 1763-1778, (2008)

23. L. Susmel, D. Taylor, Can the conventional high-cycle multiaxial fatigue criteria be reinterpreted in terms of the theory of critical distances, Struct. Durab. Health Monitor, 2, 91-108, (2006)

24. S. Fatemi, D. N. Socie, A critical plane approach to multiaxial fatigue damage including out-of-phase loading, Fatigue Fract. Eng. Mater. Struct. 11, 149-165, (1988)

25. R. N. Smith, P. Watson, T. H. Topper, A stress strain parameter for the fatigue of metal, Journal of Materials, J. Mater., 5, 767-778 (1970)

26. L. Susmel, P. A. Lazzarin, A bi-parametric w"ohler curve for high cycle multiaxial fatigue assessment, Fatigue Fract. Eng. Mater. Struct., 25, 63-78 (2002)

27. W. N. Findley, A theory for the effect of mean stress on fatigue of metals under combined torsion and axial load or bending, Engng Mat. Research Lab., Division of Engng, Brown University (1958)

28. K. Dang Van, Sur la résistance à la fatigue des métaux., Sci, Tech. L'armement, 47, 429453, (1973)

29. A. R. Kallmeyer, A. Krgo, P. Kurath, J. Engng Mat. Technology, 124, 229-237, (2002)

30. A. Carpinteri, A. Spagnoli, S. Vantadori, Multiaxial fatigue assessment using a simplified critical plane-based criterion. I, Int. J. Fatigue, 33, 969-976, (2011) 